\title{
REVIEW
}

\section{Review of Lidocaine/Tetracaine Cream as a Topical Anesthetic for Dermatologic Laser Procedures}

Tina Alster

To view enhanced content go to www.paintherapy-open.com Received: February 13, 2013 / Published online: April 6, 2013

(C) The Author(s) 2013. This article is published with open access at Springerlink.com

\section{ABSTRACT}

There are multiple different topical anesthetic options available to minimize the pain associated with cosmetic dermatologic procedures. These options, either alone or in combination, have diverse profiles for effectiveness, ease of use, application time, need for occlusion, and side effects. The lidocaine/tetracaine cream (Pliaglis ${ }^{\circledR}$, Galderma Laboratories, Texas, USA), one of the newer combination options, offers effective pain alleviation that has been evaluated in numerous clinical trials. This combination anesthetic is associated with a very favorable profile because of its ease of use and mild side effects compared to other topical local

T. Alster $(\bowtie)$

Washington Institute of Dermatologic Laser

Surgery, 1430K St NW Suite 200, Washington, D.C. 20005, USA

e-mail: talster@skinlaser.com

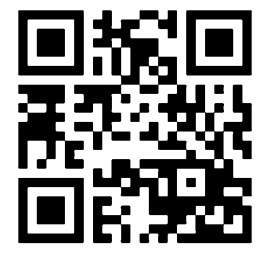

Enhanced content for this article is

available on the journal web site:

www.paintherapy-open.com anesthetics. An overview of available topical local anesthetics will be provided with an outline of clinical study characteristics and results regarding the use of lidocaine/ tetracaine cream.

Keywords: Anesthesia; Dermatologic laser procedures; Lidocaine; Pain; Pliaglis; Tetracaine; Topical administration; Treatment

\section{INTRODUCTION}

Due to the popularity of cosmetic procedures, there is a growing need for topical anesthetics that can be used in an outpatient setting for various dermatological therapies. Injectable anesthetics can be viewed as painful and, thus, difficult to administer in patients who are afraid of needles. One of the benefits of topical local anesthetics is effective anesthesia for certain procedures with little to no systemic exposure to the agent. However, topical anesthetics are often limited by the need for occlusive dressings in order to enhance their epidermal penetration $[1,2]$. In addition, inadequate or non-uniform placement of anesthesia over the skin area, 
messy application, and need for a lengthy application period are other limitations of topical anesthetics [1, 2]. Ideally, topical anesthetics must be able to penetrate the relatively impermeable barrier of the stratum corneum and have minimal systemic absorption [3, 4].

There are various forms of local anesthetics. Each has a molecular structure that includes an aromatic ring, an intermediate chain, and an amine group. All local anesthetics are lipophilic and soluble in water [5]. Lipophilia permits diffusion of the anesthetic through nerve cell membranes, and water solubility allows preparation, storage, and administration of the agent [5]. The intermediate chain determines the division of anesthetics into two classes: amide- or ester-containing [6]. Common amide anesthetics include lidocaine, prilocaine, etidocaine, and bupivacaine [6]. Procaine, benzocaine, and tetracaine are common ester anesthetics [6]. Amide anesthetics are often preferred over ester anesthetics because of the allergic reactions associated with the ester class [6, 7]. Most topical anesthetics consist of lidocaine, prilocaine, tetracaine, or a combination of them [8, 9]. All local anesthetics except cocaine have vasodilatory effects [7]. An ideal topical anesthetic agent would be easy to apply, show high clinical effectiveness over a short time period, exert its effect on intact skin without systemic effects, and cause nominal pain or discomfort during treatment with minimal to no side effects $[1,3]$. There have been many different formulations developed to meet these characteristics [3]. An overview of available topical local anesthetics will be provided with an outline of clinical study characteristics and results regarding the use of lidocaine/tetracaine cream for dermatologic laser procedures.

\section{METHODS}

A literature search was conducted in MEDLINE and EMBASE for articles published in English pertaining to topical anesthetics. The search terms included "Pliaglis," "peel," "lidocaine," "tetracaine," "local," "topical," "laser," "dermatology," and "anesthetics". Individual articles identified as relevant and pertaining to the scope of this review were identified and summarized.

\section{Current Treatments}

Various topical anesthetics are available overthe-counter or upon a physician's prescription. General anesthetics have evolved from "coca," used by the Incas, to the use of cocaine. In the late nineteenth century and early twentieth century, the isolation and use of ester anesthetics as topical agents came into practice [5]. The high degree of allergic reactions and severe side effects associated with this class favored use of the amide class over these first options. As a consequence, several formulations contain lidocaine (the most commonly used topical amide anesthetic), but each one differs in administration, length of duration, cost, and concentration [6]. Many of the over-the-counter topical anesthetics contain lidocaine $(4 \%$ or $5 \%$ ) as a monotherapy or in combination with another topical anesthetic. Some examples of these over-the-counter topical products include LMX $^{\circledR} \quad$ (Ferndale Laboratories, Ferndale, MI, USA), Betacaine ${ }^{\circledR}$ enhanced Gel 4 (Tiberius, Inc., Tampa, FL, USA), and Topicaine ${ }^{\circledR} 4 \%$ (ESBA Laboratories, Inc., Jupiter, FL, USA) [6]. Tetracaine ( $4 \%$ ) has also been formulated and distributed as a monotherapy. It is more lipophilic than lidocaine and accumulates in the stratum corneum where it slowly diffuses, 
prolonging its duration and limiting systemic uptake [10].

The most common topical anesthetic, and often regarded as the gold standard by which others are compared, is EMLA ${ }^{\circledR}$ cream (Astra Pharmaceuticals, Westborough, MA, USA) [6, 11]. EMLA cream is an emulsion in which the oil phase is a eutectic mixture of $2.5 \%$ lidocaine and $2.5 \%$ prilocaine [1]. Another common topical lidocaine formulation, LMX, uses liposomes as the delivery method for improved penetration and for sustained release of the anesthetic agent (4\% lidocaine) even after the cream is removed from the skin $[6,11]$. Both of these topical treatment options are effective at relieving pain, but they require lengthy application times and occlusion, and may provide inadequate anesthesia at peripheral skin margins $[11,12]$. EMLA cream has proven to have anesthetic efficacy in several clinical trials involving such dermatologic procedures as cryotherapy, pulsed dye laser treatment, and debridement of leg ulcers [13$16]$.

Lidocaine/tetracaine cream (Pliaglis $^{\circledR}$, Galderma Laboratories, Texas, USA) is the first Food and Drug Administration (FDA)-approved stable compounded mixture of $7 \%$ lidocaine and $7 \%$ tetracaine cream [11]. Lidocaine/ tetracaine cream has the highest approved concentrations of lidocaine and tetracaine that has also proven to be safe and effective in producing local dermal anesthesia for dermatological procedures [12]. Its novel vehicle functions as a self-occluding creamthe product is applied as a cream that dries to a pliable membrane upon exposure to air $[6,12]$. This unique formulation allows for easy application, removal, and access to areas deemed difficult for other topical anesthetics. The lidocaine/tetracaine cream was available on the market for approximately a year and a half before it was voluntarily removed due to manufacturing issues unrelated to safety at a third party contract manufacturer. It has recently been made available again with Galderma Laboratories responsible for its manufacture.

\section{Mode of Action}

Local anesthetics function by preventing the depolarization of nerve cells by the inhibition of sodium channels and thereby blocking nerve conduction [5, 6]. Myelinated A nerve fibers are typically responsible for regulating pain and temperature [5]. Sodium ion flux across the nerve cell membrane allows for the depolarization of the cell and the resultant activation of the nerve. Epinephrine or another adrenergic agonist is often added to the local topical agent in order to prolong the anesthetic effects by increasing vasoconstriction and decreasing the rate of systemic absorption [11]. Vasoconstriction increases the duration of time the agent is in contact with the nerve fibers; thus, prolonging the anesthetic effects [11].

\section{Dose, Administration, and Duration}

Lidocaine/tetracaine cream is a topical local anesthetic cream composed of equal parts of lidocaine (7\%) and tetracaine (7\%) that forms a pliable membrane on the skin when exposed to air. It is indicated for use on intact skin in adults to provide topical local analgesia for superficial dermatological procedures. A flat-surfaced tool, such as a metal spatula or tongue depressor, can be used to apply a uniform thin $(1 \mathrm{~mm})$ layer of the cream.

Lidocaine/tetracaine cream has been demonstrated to be safe and effective with minimal side effects [3]. The median duration 
of analgesia in a clinical study of 40 subjects was $11 \mathrm{~h}$. No differences in analgesic effect have been seen for 30- and 60-min application periods [17]. The systemic absorption of lidocaine and tetracaine is directly related to both the duration of application and the surface area over which it is applied [17]. Application of $59 \mathrm{~g}$ of lidocaine/tetracaine cream over $400 \mathrm{~cm}^{2}$ for up to $120 \mathrm{~min}$ in adults produced peak lidocaine concentrations of $220 \mathrm{ng} / \mathrm{mL}$ and undetectable tetracaine plasma levels $(<0.9 \mathrm{ng}$ / $\mathrm{mL}$ ) [17]. The amount of lidocaine/tetracaine cream to be used, measured in length by a ruler, is determined by the size of the area to be treated based on a chart found in the prescribing information insert.

Most other prescribed topical anesthetics are applied to the skin and occluded with plastic wrap to enhance cutaneous absorption. The occlusion and the duration of application increase the cream's penetration and efficacy [18-20]. EMLA cream, similar to other topical anesthetics, requires 40-60 min of application under occlusion for adequate analgesic effect [21]. Analgesia is achieved to a depth of $3 \mathrm{~mm}$ after $60 \mathrm{~min}$ and a maximum depth of $5 \mathrm{~mm}$ after 120 min of EMLA exposure [5]. As such, EMLA has been shown to provide effective anesthesia for several different procedures, but requires occlusion and does not provide adequate anesthesia at peripheral skin margins [1, 13-16].

\section{CLINICAL EVALUATION: EFFICACY}

There have been numerous clinical studies that evaluated the effectiveness of lidocaine/ tetracaine cream as an anesthetic agent. One of the earliest trials evaluated its use for cutaneous laser surgery compared to placebo [2]. The blinded, randomized trial had three protocols with different anesthetic application times. In the first protocol, 30 subjects were randomized to receive placebo or lidocaine/ tetracaine cream for $60 \mathrm{~min}$. The subjects, the investigators performing the laser surgery, and an independent observer evaluated clinical effectiveness. In the second and third protocols, subjects $(n=15$, each $)$ were randomized to receive placebo or lidocaine/ tetracaine cream for 20 or $30 \mathrm{~min}$ [2]. Subjects that received the active drug had less pain compared to those who received the placebo [2]. The pain ratings on the visual analog scale (VAS) were similar for the three end points [2]. In the total subject population, $9 \%$ receiving the active drug reported inadequate pain relief compared to $66 \%$ in the placebo group [2]. Similarly, the investigators rated $75 \%$ of the lidocaine/tetracaine cream subjects with no pain and $25 \%$ for the placebo group [2].

Similar results were obtained in two randomized, double-blind, placebo-controlled studies that evaluated anesthetic effect of lidocaine/tetracaine cream for laser treatment on the legs [1]. The first study followed 60 subjects where each subject served as his or her own control receiving both lidocaine/tetracaine cream and placebo. Application sites and duration of application (30 or $60 \mathrm{~min}$ ) were randomly assigned. Thirty-minute application times were determined to be inadequate, so subsequent subjects were assigned to $60-\mathrm{min}$ application times [1]. The second study evaluated 40 subjects using 60- and 90-min application times for identical laser treatment parameters [1]. Subjects evaluated anesthetic efficacy based on a VAS scale, whereas investigators and independent observers evaluated efficacy on a separate pain scale. Study 1 subjects in the active drug group had statistically lower VAS scores compared to placebo subjects $(P=0.046)$ [1]. Investigators 
reported no pain in $50 \%$ of active drug subjects and in $33 \%$ of placebo subjects. Study 2 subjects in the active drug group also had statistically better VAS scores compared to the placebo subjects for both time points $(P=0.01$ and $P<0.001$ ) [1]. Lidocaine/tetracaine cream was found to be superior in producing anesthetic effects compared to the placebo in both studies [1]. Similar results were found in another double-blinded, randomized, placebocontrolled study with laser treatment of leg veins. Significantly less pain was noted with lidocaine/tetracaine cream use compared to placebo by subjects and investigators $(P<0.001)$ [22].

The effectiveness of lidocaine/tetracaine cream was further evaluated in a double-blind study of 20 subjects undergoing nonablative laser treatment [12]. Subjects, investigators, and independent observers assessed the anesthetic effects of lidocaine/tetracaine cream compared to placebo after a 30-min application time [12]. VAS scores were significantly lower in the treatment sites compared to placebo $(P<0.001) \quad[12] . \quad$ Investigators also noted adequate anesthesia in $95 \%$ of treatment sites compared to $20 \%$ of placebo sites [12].

Lidocaine/tetracaine cream and EMLA cream were compared in a head-to-head, double-blind study of 20 patients before ablative $\mathrm{CO}_{2}$ laser skin resurfacing, a more painful laser procedure than most [23]. Subjects were randomly assigned to receive either lidocaine/tetracaine cream or EMLA cream to the treatment areas. Subjects, investigators, and independent assessors assessed the pain relief using the VAS and other pain scales [23]. Patients who received lidocaine/tetracaine cream had lower pain scores compared to those who received EMLA cream [23]. Ninety-five percent of lidocaine/tetracaine cream subjects reported adequate pain relief compared to $20 \%$ of
EMLA cream subjects [23]. The investigators and independent assessors also observed similar results [23].

In 2005, two studies were published evaluating lidocaine/tetracaine cream use with similar findings. A randomized, double-blind, placebo-controlled study evaluated lidocaine/ tetracaine cream application for $60 \mathrm{~min}$ in 30 subjects undergoing laser-assisted tattoo removal [24]. Subjects reported significantly lower pain scores with lidocaine/tetracaine cream use $(P=0.001)$ [24]. The investigator evaluations were similar to subject observations $(P<0.001)$ [24]. Another randomized, double-blind, placebo-controlled parallel study evaluated lidocaine/tetracaine cream use for $20 \mathrm{~min}$ in 80 subjects receiving pulsed dye laser therapy [25]. Subject pain scores were significantly lower in the lidocaine/tetracaine cream group $(P=0.008)$ [25]. The investigator evaluations also favored lidocaine/tetracaine cream over placebo (all $P$ values $<0.0069$ ) [25].

Most recently, lidocaine/tetracaine cream was studied in a phase 2, double-blind, placebo-controlled study in patients undergoing laser-assisted hair removal [26]. The phase 2 study evaluated 60 subjects with doses dependening on treatment surface area. Subjects were randomized to $30-, 45-$, or $60-\mathrm{min}$ treatment groups with concurrent applications of lidocaine/tetracaine cream and placebo to treatment area halves [26]. Subjects, investigators, and independent observers rated the pain experienced by the subjects after the procedure [26]. Lidocaine/tetracaine cream subject pain evaluations were significantly lower than those of placebo $(P<0.001)$ [26]. Subjects also reported significantly greater adequate anesthesia with lidocaine/tetracaine cream compared to placebo $(P<0.001)$ [26]. The investigators and observers reported similar evaluations for both of these end points [26]. 


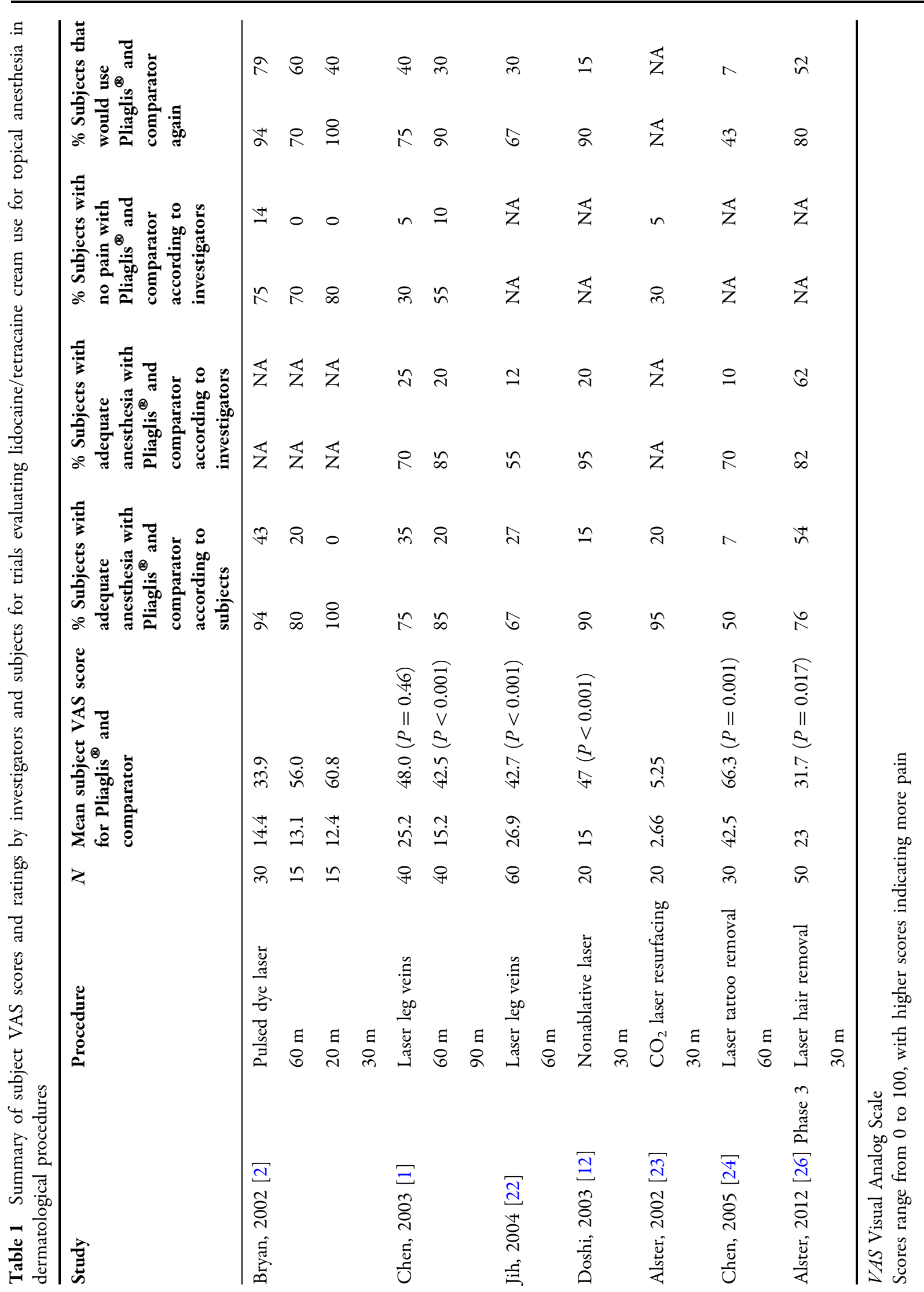


A summary of lidocaine/tetracaine cream studies for dermatologic laser procedures is presented in Table 1 [1, 2, 12, 22-26].

\section{Safety and Tolerability}

There are no set standard guidelines for the use and safety of compounded mixtures, so the need for a safe treatment profile is crucial $[5,7$, 11]. This is particularly true given the reports of systemic toxicity and four deaths associated with other topical anesthetic preparations, especially when used under occlusion with laser procedures [7, 27, 28]. Topical anesthetics are generally regarded as safe, but compounding in nonstandard doses and excessive exposure can result in dangerous results [29]. While the risk of systemic absorption of topical lidocaine is relatively low, side effects indicating systemic effect include dizziness, drowsiness, muscle twitches, seizures, respiratory distress, loss of consciousness, and cardiac arrest [6]. Allergic reactions to local anesthetics are more common with use of the ester type of anesthetics [30]. There is an additional warning for use of local anesthetics, including lidocaine/tetracaine cream, in individuals with hepatic dysfunction. The half-life of lidocaine may be increased in these subjects and those with cardiac dysfunction [17]. In addition, epinephrine, which is typically added to lidocaine preparations to extend the anesthetic effect, is associated with skin blanching [31, 32].

The typical adverse effects, including edema, erythema, and blanching, seen with EMLA cream are transient and usually localized to the application site [11]. EMLA has also been associated with methemoglobinemia, a serious condition caused by oxidation of the iron in red blood cells preventing hemoglobin from transporting oxygen. This condition is a recognized complication of other local anesthetics, particularly prilocaine [11, 33, 34].

Lidocaine/tetracaine cream is FDA-approved for adults 18 years and older, and has a welltolerated safety profile with mild and transient side effects when used as recommended $[3,11$, $30,35]$. Its most common side effects are transient skin erythema, skin discoloration, and edema [2, 3, 11]. At least one case of urticaria has been reported [30]. Although there have been no reports of methemoglobinemia with lidocaine/tetracaine cream use, it is listed as a warning for local anesthetics, including tetracaine. In addition, subjects with a known history of sensitivity to lidocaine, tetracaine, local anesthetics (amide or ester), and paraaminobenzoic acid (PABA) should not use lidocaine/tetracaine cream [17].

The systemic levels of lidocaine and tetracaine in the plasma of 36 subjects that used lidocaine/tetracaine cream were evaluated to determine the extent of systemic absorption. The pharmacokinetic profile of lidocaine and tetracaine after a single application of lidocaine/ tetracaine cream was assessed in one open label, randomized, parallel group design [35]. Subjects were randomized to one of the three groups; 30-, 60-, and 90-min application times. The plasma samples were obtained at baseline, every $30 \mathrm{~min}$ for $3.5 \mathrm{~h}$, and 5 and $7 \mathrm{~h}$ after the initial application [35]. The systemic concentrations for both lidocaine and tetracaine in all the samples were below the lower limits of quantification for the assays used (lidocaine $100 \mathrm{ng} / \mathrm{mL}$, tetracaine $5 \mathrm{ng} / \mathrm{mL}$ ) [35]. As a result, the pharmacokinetic parameters beyond maximum concentration $\left(C_{\max }\right)$ could not be determined [35]. Central nervous system toxicity for lidocaine is typically observed at $5,000 \mathrm{ng} / \mathrm{mL}$; a small number of subjects may show signs of toxicity at $1,000 \mathrm{ng} / \mathrm{mL}$ [17]. 
Tetracaine levels of toxicity are similar to lidocaine, but at lower levels-the exact numbers are unclear [17]. The use of coadministered local anesthetics toxicity levels is thought to be at least additive [17]. Providers should make sure that the doses, areas of application, and duration of application are consistent with those recommended for the intended population. The low systemic exposure and mild adverse event reports provide lidocaine/tetracaine cream with a benefit over many other available local topical anesthetics [11].

\section{CONCLUSION}

The need for continued improvement in patient pain management is growing as the number and types of outpatient dermatology procedures increase. Lidocaine/tetracaine cream offers the advantages typical of many topical anesthetics, including fast, effective pain reduction, limited systemic effects, and elimination of needles. In addition, lidocaine/tetracaine cream has several additional attributes that are unique to its formulation, including ease of application and removal, self-occluding formulation, and ability for use in skin folds.

\section{ACKNOWLEDGMENTS}

No funding or sponsorship was received for this study or publication of this article. The author would like to thank Adrienne Badeaux of Galderma, L.P. for her assistance with manuscript preparation. Dr. Alster is the guarantor for this article and takes responsibility for the integrity of the work as a whole.

Conflict of interest. Dr. Tina Alster declares no conflict of interest.
Open Access. This article is distributed under the terms of the Creative Commons Attribution Noncommercial License which permits any noncommercial use, distribution, and reproduction in any medium, provided the original author(s) and the source are credited.

\section{REFERENCES}

1. Chen JZ, Alexiades-Armenakas MR, Bernstein LJ, Jacobson LG, Friedman PM, Geronemus RG. Two randomized, double-blind, placebo-controlled studies evaluating the S-Caine Peel for induction of local anesthesia before long-pulsed Nd:YAG laser therapy for leg veins. Dermatol Surg. 2003;29: 1012-8.

2. Bryan HA, Alster TS. The S-Caine peel: a novel topical anesthetic for cutaneous laser surgery. Dermatol Surg. 2002;28:999-1003 (discussion 1003).

3. Kim WO, Song BM, Kil HK. Efficacy and safety of a lidocaine/tetracaine medicated patch or peel for dermatologic procedures: a meta-analysis. Korean J Anesthesiol. 2012;62:435-40.

4. Kang C, Shin SC. Development of prilocaine gels for enhanced local anesthetic action. Arch Pharm Res. 2012;35:1197-204.

5. Sobanko JF, Miller CJ, Alster TS. Topical anesthetics for dermatologic procedures: a review. Dermatol Surg. 2012;38:709-21.

6. Railan D, Alster TS. Use of topical lidocaine for cosmetic dermatologic procedures. J Drugs Dermatol. 2007;6:1104-8.

7. Berkman S, MacGregor J, Alster T. Adverse effects of topical anesthetics for dermatologic procedures. Expert Opin Drug Saf. 2012;11:415-23.

8. Croxtall JD. Lidocaine/tetracaine medicated plaster: in minor dermatological and needle puncture procedures. Drugs. 2010;70:2113-20.

9. Tadicherla S, Berman B. Percutaneous dermal drug delivery for local pain control. Ther Clin Risk Manag. 2006;2:99-113.

10. Russell SC, Doyle E. A risk-benefit assessment of topical percutaneous local anaesthetics in children. Drug Saf. 1997;16:279-87.

11. Alster TS. The lidocaine/tetracaine peel: a novel topical anesthetic for dermatologic procedures in adult patients. Dermatol Surg. 2007;33:1073-81. 
12. Doshi SN, Friedman PM, Marquez DK, Godlberg LH. Thirty-minute application of the S-Caine peel prior to nonablative laser treatment. Dermatol Surg. 2003;29:1008-11.

13. Mansell-Gregory M, Romanowski B. Randomised double blind trial of EMLA for the control of pain related to cryotherapy in the treatment of genital HPV lesions. Sex Transm Infect. 1998;74:274-5.

14. Ashinoff R, Geronemus RG. Effect of the topical anesthetic EMLA on the efficacy of pulsed dye laser treatment of port-wine stains. J Dermatol Surg Oncol. 1990;16:1008-11.

15. Holm J, Andren B, Grafford K. Pain control in the surgical debridement of leg ulcers by the use of a topical lidocaine-prilocaine cream, EMLA. Acta Derm Venereol. 1990;70:132-6.

16. Blanke W, Hallern BV. Sharp wound debridement in local anaesthesia using EMLA cream: 6 years' experience in 1084 patients. Eur J Emerg Med. 2003;10:229-31.

17. Galderma USA. Pliaglis prescribing information. http://www.galdermausa.com/pdf/101567000407\%20PLIAGLIS\%20Package\%20Insert.pdf. Accessed 19 Mar 2013.

18. Wahlgren CF, Quiding H. Depth of cutaneous analgesia after application of a eutectic mixture of the local anesthetics lidocaine and prilocaine (EMLA cream). J Am Acad Dermatol. 2000;42:584-8.

19. Chen BK, Eichenfield LF. Pediatric anesthesia in dermatologic surgery: when hand-holding is not enough. Dermatol Surg. 2001;27:1010-8.

20. Tahir A, Webb JB, Allen G, Nancarrow JD. The effect of local anaesthetic cream (EMLA) applied with an occlusive dressing on skin thickness. Does it matter? J Plast Reconstr Aesthet Surg. 2006;59:404-8.

21. Sawyer J, Febbraro S, Masud S, Ashburn MA, Campbell JC. Heated lidocaine/tetracaine patch (Synera, Rapydan) compared with lidocaine/ prilocaine cream (EMLA) for topical anaesthesia before vascular access. Br J Anaesth. 2009;102:210-5.

22. Jih MH, Friedman PM, Sadick N, Marquez DK, Kimyai-Asadi A, Goldberg LH. 60-minute application of S-Caine Peel prior to $1,064 \mathrm{~nm}$ long-pulsed Nd:YAG laser treatment of leg veins. Lasers Surg Med. 2004;34:446-50.

23. Alster TS, Lupton JR. Evaluation of a novel topical anesthetic agent for cutaneous laser resurfacing: a randomized comparison study. Dermatol Surg. 2002;28:1004-6 (discussion 1006).

24. Chen JZ, Jacobson LG, Bakus AD, et al. Evaluation of the $\mathrm{S}$-Caine peel for induction of local anesthesia for laser-assisted tattoo removal: randomized, double-blind, placebo-controlled, multicenter study. Dermatol Surg. 2005;31:281-6.

25. Schecter AK, Pariser DM, Pariser RJ, et al. Randomized, double-blind, placebo-controlled study evaluating the lidocaine/tetracaine patch for induction of local anesthesia prior to minor dermatologic procedures in geriatric patients. Dermatol Surg. 2005;31:287-91.

26. Alster T, Garden J, Fitzpatrick R, Rendon M, Sarkany M, Adelglass J. Lidocaine/tetracaine peel in topical anesthesia prior to laser-assisted hair removal: PhaseII and Phase-III study results. J Dermatol Treat. 2012;1-4. doi:10.3109/09546634.2012.715240

27. Hahn IH, Hoffman RS, Nelson LS. EMLA-induced methemoglobinemia and systemic topical anesthetic toxicity. J Emerg Med. 2004;26:85-8.

28. Rincon E, Baker RL, Iglesias AJ, Duarte AM. CNS toxicity after topical application of EMLA cream on a toddler with molluscum contagiosum. Pediatr Emerg Care. 2000;16:52-4.

29. Kaweski S; Plastic Surgery Educational Foundation Technology Assessment Committee. Topical anesthetic creams. Plast Reconstr Surg. 2008;121:2161-5.

30. Channual J, Wu JJ, Zachary CB. Localized contact urticaria caused by lidocaine/tetracaine peel. Arch Dermatol. 2009;145:499-500.

31. Kundu S, Achar S. Principles of office anesthesia: part II. Topical anesthesia. Am Fam Phys. 2002;66: 99-102.

32. Irsfeld S, Klement W, Lipfert P. Dermal anaesthesia: comparison of EMLA cream with iontophoretic local anaesthesia. Br J Anaesth. 1993;71:375-8.

33. Nishimura K. Methemoglobinemia due to local anesthetics. Osaka City Med J. 1971;17:25-42.

34. Guay J. Methemoglobinemia related to local anesthetics: a summary of 242 episodes. Anesth Analg. 2009;108:837-45.

35. Ogden L, Love G, Basta S. Systemic exposure to lidocaine and tetracaine is low after an application of a lidocaine $7 \%$-tetracaine $7 \%$ peel in adults. Int $\mathrm{J}$ Dermatol. 2008;47:87-90. 